\title{
Frequency Diverse Scanning for Aperiodic (Random) Antenna Arrays
}

\author{
Timi Adeyemi and Kris Buchanan \\ Information Operations Warfare \\ Space and Naval Warfare Systems Center (SSC) Pacific \\ San Diego, USA \\ timi.adeyemi@navy.mil,kris.buchanan@navy.mil
}

\begin{abstract}
Network centric warfare, swarming and other multi-static radar applications are of increasing interest in defense and commercial applications. Using a distributed sensing network to collaboratively beamform improves electronic scan capabilities, enhances resolution, range and bandwidth, when compared with traditional phased arrays. We investigate a new architecture for an aperiodic (random) array configuration called frequency diverse array, which gives rise to range, time, and angle-dependent scanning without the use of phase shifters.
\end{abstract}

Keywords- Random array, frequency diverse array, opportunistic array, cooperative beamforming, distributed beamforming, wireless sensor network

\section{INTRODUCTION}

Limitations of periodic phased antenna arrays are well known. Recent studies [1], [2] have applied frequency diversity to periodic arrays showing many potential benefits including clutter and interference suppression, flexible scan, multipath mitigation, and increased bandwidth due to range and angle dependence. However, traditional periodic arrays may not be the optimal configuration for frequency diverse arrays [3]. By applying frequency diversity to random arrays, we find advantages including increased resolution, increased beam scanning flexibility, and non-traditional geometrical element placement.

\section{ANALYSIS OF RANDOM ARRAYS AND FREQUENCY DIVERSITY}

\section{A. Random Array Setup}

We assume a uniformly aperiodic volumetric random array with perfect phase information bound to a Euclidean domain [1] as in Fig. 1. The element distance $R_{n}(\theta, \phi)$ in (1) is defined as the Euclidean distance between $P_{n}$ and $P$.

$$
R_{n}\left(\theta, \phi \mid r_{n}, \theta_{n}, \phi_{n}\right)=r \sqrt{1+\left(r_{n} / r\right)^{2}-2 r_{n} \cos \psi_{n} / r}
$$

If $P$ is assumed to reside in the far field of the array and $P_{n}$ is the location of $n$th element in the spherical bound, the common approximations are a first order approximation for the magnitude $R_{n} \approx r$ and second order approximation for phase $R_{n}$ $\approx r-r_{n} \cos \psi_{n}$.

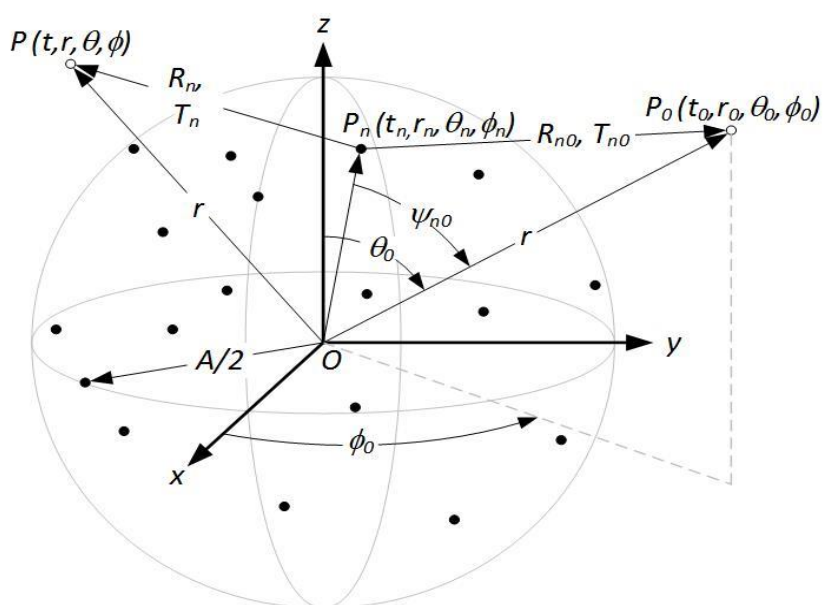

Fig. 1. Random distribution of elements in a spherical volume

This can be found from the binomial expansion (2) or by linking the direction vectors from the origin and from $P_{n}$ to $P$ by the direction cosine in (3) where $\hat{r}_{n}$ and $\hat{r}$ are the unit radial vectors for $P_{\mathrm{n}}$ and $P_{0}$ respectively. The array factor is found as

$$
\begin{gathered}
R_{n} \approx \sum_{n=0}^{N} r\left(\begin{array}{c}
1 / 2 \\
n
\end{array}\right)\left(\left(\frac{r_{n}}{r}\right)^{2}-\frac{2 \cos (\psi) r_{n}}{r}\right)^{n} . \\
\cos \psi_{n}=\hat{r}_{n} \cdot \hat{r}=\sin \theta_{n} \sin \theta \cos \left(\phi-\phi_{n}\right)+\cos \theta_{n} \cos \theta \\
F\left(r, \theta, \phi \mid r_{n}, \theta_{n}, \phi_{n}\right) \approx \frac{1}{N} \sum_{n=1}^{N} e^{j k A \tilde{r}_{n}\left(\cos \psi_{n}-\cos \psi_{n o}\right)}=\frac{1}{N} \sum_{n=1}^{N} e^{j \Psi} \\
\Psi=k A r_{n}\left(\cos \psi_{n}-\cos \psi_{n o}\right)
\end{gathered}
$$

(4) where $N$ is the number of elements in the array, $k$ is the wavenumber, $A$ is the diameter of the aperture size, $\tilde{r}_{n}$ is the random variable location normalized by $A, \cos \psi_{n}$ is the directional cosine of the $n^{\text {th }}$ element $r_{\mathrm{n}}$ and $\cos \psi_{n 0}$ is the difference between the directional cosine of $r_{\mathrm{n}}$ and the directional cosine of the target at $P_{0}$. 


\section{B. Frequency Diversity for Random Array}

Applying frequency diversity to the array, each element is assigned a unique frequency $f_{\mathrm{n}}$. This frequency is an offset $\left(\Delta f_{\mathrm{n}}\right)$ of the operating frequency $\left(f_{0}\right)$, where we assume $\Delta f_{\mathrm{n}}<<$ $f_{0}$. We can steer the array by taking the phase difference between the $n^{\text {th }}$ element $\Psi_{\mathrm{n}}(5)$ and the reference element $\Psi_{0}$ (5). We note that this difference, $\Delta \Psi$ in (6), contains phase steering terms not seen in the traditional phased array, from which we obtain additional degrees of freedom.

$$
\begin{aligned}
& \Psi_{0}=k R_{0}=2 \pi R_{0} / \lambda \approx 2 \pi f_{0}\left(r_{0}\right) / c \\
& \Psi_{n}=k R_{n} \approx 2 \pi f_{n} R_{n}(\theta, \phi) / c=2 \pi\left(f_{0}+\Delta f_{n}\right)\left(r_{0}-r_{n}\left(\cos \psi_{n 0}\right)\right) / c \\
& f_{n}=\left(f_{0}+\Delta f_{n}\right) \\
& R_{n}(\theta, \phi) \approx r_{0}-r_{n} \cos \psi_{n} \\
& \left.\Delta \Psi\right|_{\Psi_{0}-\Psi_{n, n 0}}=\frac{2 \pi}{c}\left(\left\{f_{0} r_{n}\left(\cos \psi_{n}\right)\right\}+\Delta f_{n} r_{n}\left(\cos \psi_{n 0}\right)-\Delta f_{n} r_{0}\right)=0
\end{aligned}
$$

The array factor of (4) is satisfied by finding the radiation intensity $(U)$ in (7), solving for $\Delta f_{\mathrm{n}}$ and setting $r=r_{0}$ in (8), noting that $F$ is the array factor and $F^{*}$ is its complex conjugate.

$$
\begin{gathered}
U=F F^{*}\left(\left(e^{j k r}\right)\right)\left(\left(e^{-j k r}\right)\right)=1 \\
e^{j \frac{2 \pi}{c}\left(f_{0} r_{n}\left(\cos \psi_{n}\right)-\Delta f_{n}\left[r_{n}\left(\cos \psi_{n 0}\right)-r\right]\right)}
\end{gathered}
$$

\section{GRAPHICAL REPRESENTATION OF FREQUENCY DIVERSE BEAMSTEERING APPLIED TO A RANDOM ARRRY}

In [4] it is demonstrated that the expected value across the unit interval $[-1,1]$ for a random variable $x$ in any volumetric distribution provides the characteristic functions described in (9).

$$
\bar{U}=\frac{1}{N}+\left(1-\frac{1}{N}\right)|\Lambda|^{2}
$$

From this, the mean-valued radiation intensity $(\bar{U})$ can be found where $|\Lambda|^{2}$ is the characteristic function of the array factor given in (4) and $\mathrm{N}$ is the number of elements. The first sidelobe level and main lobe of the characteristic function have deterministic properties. As a consequence, the same methods used in aperture theory may be applied to random arrays [4]. Setting $r_{0}=100 \mathrm{~km}$ in (6), we plot the mean-valued radiation intensity of a spherical random array (SRA) in Fig.2. The pattern converges to the expected valued due to the high element count of 100. In the top left of Fig. 2 we see that the pattern contains a main beam and low sidelobes at $r_{0}$. The high directivity of the main beam can be shown to be proportional to the number of elements [5]. At $r=1000 \mathrm{~km}$ and $10 \mathrm{~km}$, beams are pointed in multiple directions indicating that the pattern is a function of distance. Additionally, the three plots on the left column, which are plots of the spherical bound of the radiation intensity at a distance $r$ from the reference point, show that the pattern is a function of angle.
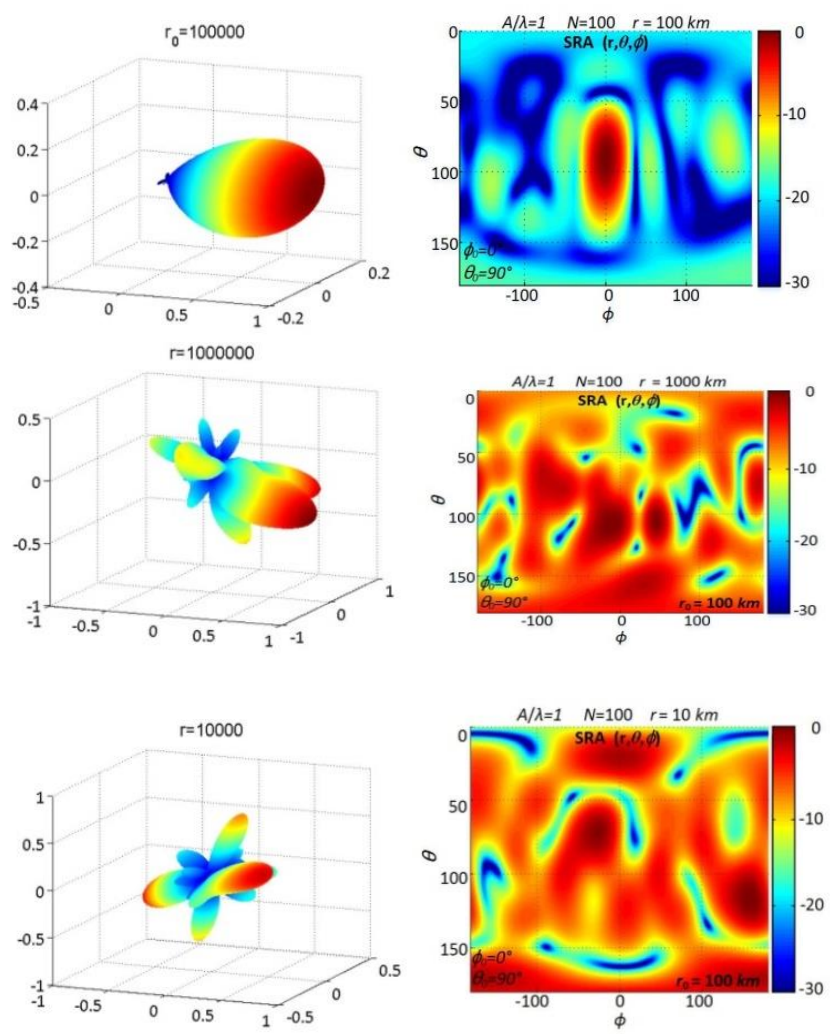

Fig. 2.Spherical random array steered at $r_{0}$ in top row, $r_{0} \times 10$ in middle row, and $r_{0} / 10$ in bottom row. The three-dimensional radiation patterns are shown in the left column and topological images in the right column.

\section{CONCLUSION}

We analyzed the frequency diverse antenna performance for a spherical bound random array. We conclude that frequency diversity can provide additional degrees of freedom to beamsteer as a function of angle and range when applied to a random array. The aperiodic nature of the array increases resolution which enhances beamsteering ability. These inherent benefits can be incorporated into new collaborative beamforming designs.

\section{REFERENCES}

[1] W.Wang, H. Shao, J. Cia., "Range-Angle-Dependent Beamforming by Frequency Diverse Array Antenna," International Journal of Antennas and Propagation, vol. 2012, pp. 1-10, 2012.

[2] P. Antonik, An Investigation of a Frequency Diverse Array, London: University College London, 2009.

[3] W. Wang, "Frequency Diverse Array Antenna: New Opportunities," IEEE Antennas and Propagation Magazine, vol. 57, no. 2, pp. 145-152, 2015.

[4] K. Buchanan, Theory and Applicaions of Aperiodic (Random) Phased Arrays, College Station: Texas A\&M University, 2012

[5] Y. Lo, "A mathematical theory of antenna arrays with randomly spaced elements," IEEE Trans. Antennas Propagation, vol. 12, pp. 257-268, 1964. 